\title{
MOLECULAR PROXIMITY EFFECTS IN TWO-PHOTON ABSORPTION
}

David L. Andrews and Kevin P. Hopk ins

School of Chemical Sciences, University of East Anglia, Norwich NR4 7TJ, England,

\begin{abstract}
Under laser irradiation, the mutual interaction between molecules is shown to lead to a cooperative nonlinear process which results in entirely new features in the absorption spectrum. In the quantum electrodynamical formalism the coupling between molecular centres, induced by molecular proximity rather than collision, is mediated by means of virtual photon coupling and allows transitions which are forbidden in the absence of such interactions. The manifestation of circular dichrolsm, potential methods of observation and specific molecular examples are discussed. Attention is drawn to a number of possible resonance enhancement mechanisms and to the dependence of the absorption rate on molecular separation.
\end{abstract}

\section{INTRODUCTION}

Standard methods of molecular quantum electrodynamics have been employed to study interactions between molecular centres which can lead to cooperative twophoton absorption. This nonlinear absorption process results from molecular proximity effects rather than collisions. The two chemical centres, which may be either distinct chromophores in a single molecule, or separate molecules, undergo cooperative excitation through two-photon absorption induced by intense laser light. Two cases are of interest.

In the first, the interaction between two non-identical centres provides the mechanism for energy exchange such that an overall process

$A+B+2 h \omega \rightarrow A^{*}+B^{*}$

can take place even when the individual transitions $A \rightarrow A^{*}$ and $B \rightarrow B^{*}$ are forbidden on energy grounds, as illustrated in Fig. 1. For this cooperative process to be experimentally observable, $\omega$ must be chosen to 11 e in a region where nelther A nor B displays absorption, and we thus have

$h \omega=k\left(E_{\alpha O}+E_{B O}\right)$,

$h \omega \neq E_{\alpha O}, E_{\infty O}$.

0022-2860/88/\$03.50 (C) 1988 Elsevier Science Publishers B.V. 
This cooperative process can take place by one of two mechanisms, in which elther (a) one laser photon is absorbed by each molecule, or (b) both are absorbed by one molecule. In each case, the energy mismatch for the molecular transitions is transferred between molecules by means of a virtual photon.

In the second case of interest, the two centres have identical composition, and are cooperatively excited by two-photon absorption from two laser beams with differing frequencies, $\omega_{1}$ and $\omega_{2}$. This process can be represented by the equation

$A+A+h \omega_{1}+h \omega_{2} \rightarrow A^{*}+A^{*}$,

for which the energetics are shown in Fig. 2. Again, the frequencles $\omega_{1}$ and $\omega_{2}$ are chosen in a region where single-photon absorption cannot lead to the excitation of either centre. Thus we have;

$H\left(h \omega_{1}+h \omega_{2}\right)=E_{\alpha a_{1}}$

$h \omega_{1}, h \omega_{2} \neq E_{\alpha 0}$.

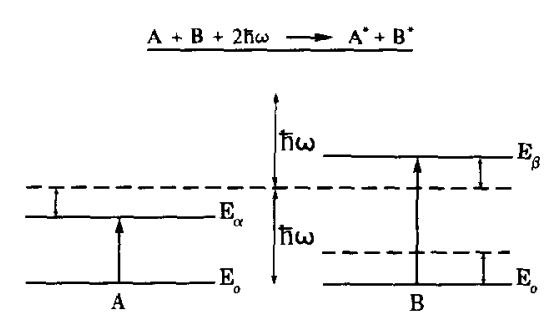

(a)

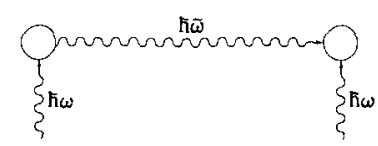

$\bar{\omega}=\Delta \mathbf{E} / \bar{\kappa}$

(b)

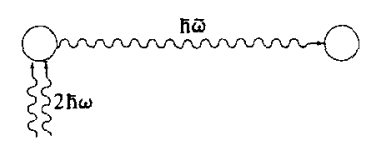

$\widetilde{\omega}=\omega-\Delta \mathrm{E} / \mathrm{h}$

Figure 1: Single-beam cooperative two-photon absorption

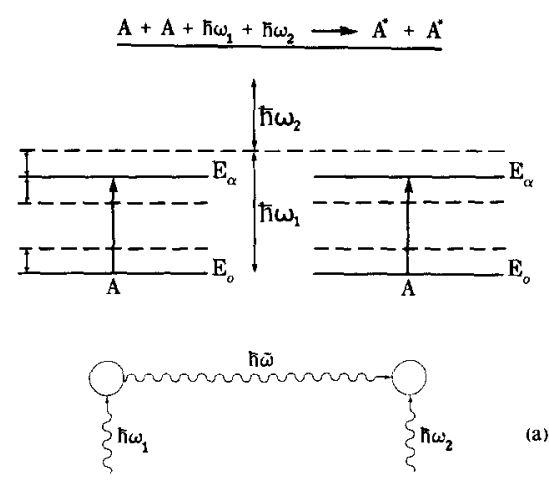

$\check{\omega}=\Delta \mathrm{E} / \hbar$

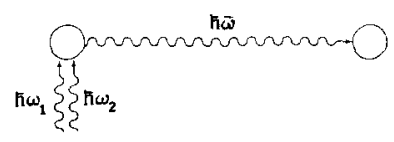

(b)

$\bar{\omega}=\omega_{1}+\Delta \mathbf{E} / \hbar$ 
The former relation (5) Indicates the fact that from a phenomenological point of vlew, this cooperative process has the characteristics of mean-frequency absorption. Once again two mechanisms can produce the effect as shown in Fig. 2 . Thus there are total of four distinct absorption routes to be considered. A detalled study of each of these is beyond the scope of this present work and, therefore, only an outline of the calculational procedure is given, along with a discussion of some of the interesting features that arise. For a complete and detalled analysis the reader is referred to a recent series of papers (refs. 1-4).

\section{QUANTUM ELECTRODYMAMICAL CALCULATIONS}

In this study, the analysis of molecular proximity effects in two-photon absorption is based on the methods of molecular quantum electrodynamics (ref, 5). Although results are calculated within the electric-dipole approximation for each molecular centre, the detailed analysis of the coupling provides results equivalent to the Inclusion of higher order multipole terms for the pair.

The rate of cooperative absorption is determined by the Fermi Golden Rule, (ref. 6), using fourth-order time-dependent perturbation theory. The oppropriate matrix element can be constructed with the aid of time-ordered-diagrams, of which there are 96 to be taken into consideration. For mechanism (a), only diagrams such as that shown in Fig. $3(\mathrm{a})$, in which two real photons are absorbed at different molecular centres, contribute. Here the virtual photon conveys the energy mismatch from one molecule to the other. In mechanism (b), represented schematically by time-ordered-diagrams such as that shown in Fig. $3(\mathrm{~b})$, both real photons are

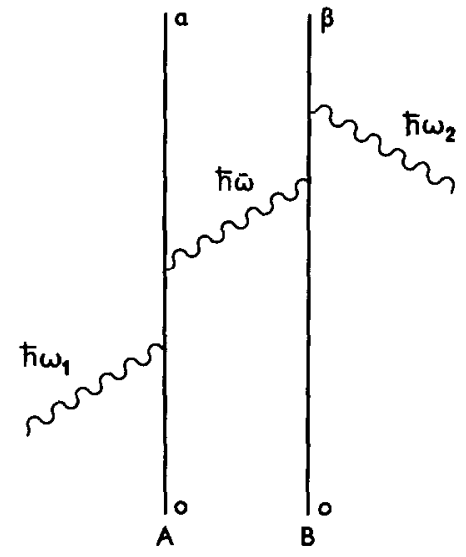

(a)

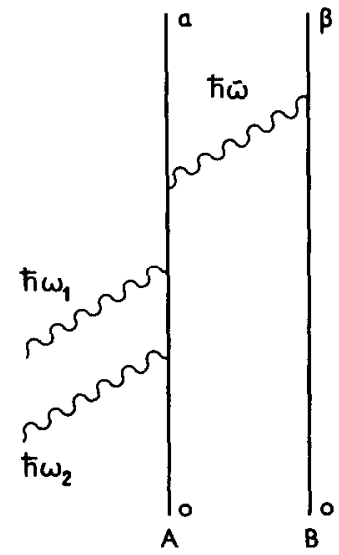

(b)

Figure 3: Typlcal time-ordered-diagrams for cooperative two-photon absorption 
absorbed at one centre, which necessitates excitation of its partner by a single virtual photon interaction. This fundamental difference between the two absorption routes is of prime significance in any consideration of selection rules.

\section{SELECTION RULES}

The two mechanisms, (a) and (b), by means of which each cooperative process may occur are associated with quite distinct selection rules. Because the virtual photon couples with each molecule by the same electric-dipole coupling as the laser photons, mechanism (a) requires for both transitions to be allowed by two-photon selection rules, whilst mechanism (b) is permitted if one transition is allowed by the faniliar one-photon selection rules. For example, in a pair of centrosymmetric species, mechanism (a) allows for both transitions to preserve parity ( $g \leftrightarrow g, u \leftrightarrow u$ ), but mechanism (b) results in parity reversal ( $u \leftrightarrow 8$ ) at each centre. Therefore in such systems, only one mechanism can be operative for any given cooperative transition.

\section{RESONANCE EHHANCEMENT}

There are several possibllities for obtaining resonance enhancement of these processes. For the single-frequency excltation case 1llustrated in Fig. 1, resonance amplification of the absorption rate will be observed if the participating molecules have energy levels close to any of the positions indicated by dashed lines on the energy level diagram. However, a molecule possessing an energy level close to the lowest of these three resonance conditions will be of most interest since energy levels at both the other indicated positions can be populated through a single-photon absorption process. For the two-beam case illustrated in Fig. 2 , rate-enhancement will again occur if the molecule concerned has energy levels at any of the positions indlcated; in this case the facility to tune laser frequencies offers much greater likelihood of being able to exploit one of the resonance conditions. Again the lowest of these resonance levels is of greatest interest since the upper levels can be populated via single-photon absorption. The successful exploitation of these resonance enhancement conditions might typically be expected to produce an increase in the absorption rate by several powers of ten.

\section{CIRCULAR DICHROISM}

Both of the processes described here can produce circular dichroism in special cases even if the centres involved are not inherently optically active. In the case of single-beam laser excitation (FIg. 1), circular dichroism is manifest if $A$ and $B$ are achtral chromophores dissymmetrically placed in a large molecule or van der Wals dimer, as in the traditional two-group model of optical activity, (ref. 7 ). 
In the two-beam case (Fig. 2) circular dichrolsm can persist even if the two centres freely tumble with respect to one another. This effect arises if one of the laser beams has a fixed polarisation. Since this confers chlrality upon the two-molecule system, circular dichroism with respect to the handedness of the second laser bean can be observed.

\section{ISCUSSION}

We have calculated the dependence of the rate of cooperative two-photon absorption on the separation of the absorbing species. In the region referred to as the near-zone, where the distance between the molecular centres, $R$, is comparable with molecular dimensions, the cooperative absorption process has a straightforward $\mathrm{R}^{-5}$ dependence. However, in the region known as the far-zone, where the absorbing centres are separated by distances which are large compared with optical wavelengths, an inverse-square dependence on $R$ is found. Examining the time-ordered-diagrams of Fig. 3-4, and considering each mechanism in the extreme far-zone. (where the two absorbing centres are far enough apart that the interaction can be considered to be mediated by a real photon), mechanism (a) can be regarded as Raman scattering by the molecular centre A followed by two-photon absorption at $B$. Similarly, mechanism (b) in the long-range limit resembles nonlinear (hyper-Raman) scattering at A, with single-photon absorption at B. Both of these Raman processes, linear and non-linear, are well known to have an inversesquare dependence on $R$, in agreement with the results obtained here.

An illustration of cooperative two-photon absorption can be provided by considering the photochemistry of a mixture of formaidehyde $\left(\mathrm{H}_{2} \mathrm{CO}\right)$ and deuterioformaldehyde $\left(D_{z} C O\right)$. These molecules have absorption. lines, (associated with the 2!4: transtition), at 30340.15 and $30147.62 \mathrm{~cm}^{-1}$

respectively. Irradiation at the mean of the two frequencles should result in the simulteneous excitation of both specles:-

$\mathrm{H}_{2} \mathrm{CO}+\mathrm{D}_{2} \mathrm{CO} \stackrel{2 \mathrm{h \omega}}{\rightarrow} \mathrm{H}_{2} \mathrm{CO}^{*}+\mathrm{D}_{2} \mathrm{CO}^{*}$

This would certainly need to be a cooperative process if the bandwidth of the laser source were sufficlently narrow to ensure that neither species could be independently excited through conventional single-photon absorption. Evidence for this proposed reaction mechanism should then be provided by the detection of the decomposition product, carbon monoxide, which results from the autodissociation reactions :-

$\mathrm{H}_{2} \mathrm{CO}^{*} \quad \rightarrow \quad\left\{\begin{array}{l}\mathrm{H}_{2}+\mathrm{CO} \\ \mathrm{H}+\mathrm{H}+\mathrm{CO}\end{array}\right.$ 
$\mathrm{D}_{2} \mathrm{CO} * \quad \rightarrow \quad\left\{\begin{array}{l}\mathrm{D}_{2}+\mathrm{CO} \\ \mathrm{D}+\mathrm{D}+\mathrm{CO}\end{array}\right.$

The photodynamics of charge transfer reactions have been studied in some depth. Setser et al (ref. 8) have performed studies on a gaseous mixture of xenon and chlorine passed slowly through a laser fluorescence cell. The proposed reaction mechan ism

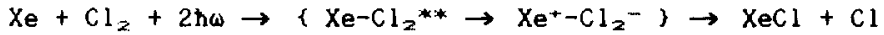

was attributed to excitation from the van der Wals ground state potential to the ion-pair potential via configurational interaction of the $\mathrm{Xe}$ and $\mathrm{Cl} \mathrm{l}_{2}$, 1.e. the excitation results from molecular proximity of the two absorbing species. This mechanism, eqn. 10, is further corroborated by Apkarian et al. (ref. 9) who have extended the study to charge transfer reactions in rare gas solids (ref, 10). It has also been shown to be the predominant reaction route in the case of $\mathrm{Xe}_{\mathrm{Cl}} \mathrm{Cl}_{2}$ van der Wals complexes generated in seeded molecular beams (ref, 11).

In conclusion, theory shows that quantum electrodynamical coupling of molecular centres in close proximity should result in cooperative two-photon transitions. The effect should be manifest through the absorption of laser light at a mean frequency where neither one- nor two-photon absorption processes nornally occur, and may result in novel photochemical behaviour.

\section{ACKNOWLEDCHMENT}

K.P.H. gratefully acknowledges $f$ inancial support from the Science and Engineering Research Council.

\section{REFEREHCES}

1. D.L. Andrews and M.J. Harlow, J. Chem. Phys., 78, (1983), 1088-1094.

2. D.L. Andrews and M.J. Harlow, J. Chem. Phys, , 80, (1984), 4753-4760.

3. D.L. Andrews and K.P. Hopkins, J, Chem. Phys., 86, (1987), 2453-2459.

4. D.L. Andrews and K.P. Hopkins, in preparation.

5. D.P. Crais and T. Thirunamachandran, Molecular Quantum Electradynamics, Academic, London, 1984

6. E. Fermi, Nuclear Physics, University of Chicago, Chicago, 1950.

7. L. D. Barron, Molecular Light Scattering and Optical Activity, Cambridge University Press, Cambridge, 1982.

8. J.K. Ku, G. Inoue and D.W. Setser, J. Phys. Chem., 87, (1983), 2989-2993.

9. L. Wiedemen, M.E. Fajardo and V.A. Apkerian, Chem. Phys. Letts, 134, (1987), 55-59.

10. M.E. Fajardo and V.A. Apkarian, J. Chem. Phys., 85, (1986), 5660-5681.

11. M. Biovineau, J. leCalve, M. C. Castex and C. Jouvet, Chem. Phys. Letts, 128, (1986), 528-531. 